\title{
Diagnósticos de enfermagem documentados para pacientes de clínica médica*
}

\author{
DOCUMENTED NURSING DIAGNOSES FOR MEDICAL CLINIC PATIENTS
}

DIAGNÓSTICOS DE ENFERMERÍA DOCUMENTADOS PARA PACIENTES DE CLÍNICA MÉDICA

\author{
Cassiana Mendes Bertoncello Fontes ${ }^{1}$, Diná de Almeida Lopes Monteiro da Cruz $^{2}$
}

\section{RESUMO}

Este artigo relata estudo descritivo, de análise de registros, dos diagnósticos de enfermagem documentados após três meses da implementação da classificação da NANDA-I no HUUSP e propõe resultados e intervenções para os três diagnósticos mais freqüentes. A amostra de conveniência, de $34 \%$ das internações no mês, constituiu-se de 30 prontuários de pacientes internados na Clínica Médica em agosto de 2004 (60\% mulheres;

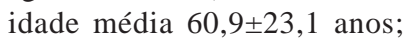
média de internação $=5,8 \pm 2,7$ dias). Os diagnósticos documentados nos prontuários, no primeiro dia de internação, foram manualmente transcritos e analisados segundo freqüências. Foram documentados 144 diagnósticos (31 categorias diagnósticas); com média de 4,8( $\pm 4,0)$ diagnósticos por paciente (variação = 1 a 10) e os mais freqüentes foram: Dor aguda (66,7\%), Integridade tissular prejudicada (63,3\%), Desobstrução ineficaz de vias aéreas (43,3\%), Risco para integridade da pele prejudicada (36,7\%) e Integridade da pele prejudicada (33,3\%). São apresentadas propostas de resultados e intervenções para os três mais freqüentes.

\section{DESCRITORES}

Diagnóstico de enfermagem. Classificação.

Medicina interna.

\section{ABSTRACT}

This paper reports a descriptive study, based on nursing records, of nursing diagnoses documented three months after the implementation of the NANDA-I classification at the University of São Paulo’s Hospital Universitário (HU-USP) and proposes outcomes and interventions for the 3 most frequent diagnoses. The convenience sample (34\% of the month's admissions) consisted of 30 charts of patients admitted in the Medical Clinic in August of 2004 (60\% female, average age $60.9 \pm 23.1$ years, mean length of stay $=5.8 \pm 2.7$ days). The diagnoses documented on the admission day were manually transcribed from the charts and analyzed according to their frequency. There were 144 diagnoses (31 categories), with an average of $4.8 \pm 4.0$ diagnoses per patient (range $=1-10)$. The most frequent were: acute pain (66.7\%), impaired tissue integrity (63.3\%), ineffective airway clearance (43.3\%), risk of impaired skin integrity (36.7\%), and impaired skin integrity (33.3\%). The proposed outcomes and interventions are presented.

\section{RESUMEN}

Este artículo relata un estudio descriptivo, de analice de registros, de los diagnósticos de enfermería documentados tres meses después de la implementación de la clasificación de la NANDA-I en el HU-USP. La muestra de conveniencia (34\% de las internaciones en el mes) constituyó de 30 prontuarios de pacientes internados en la Clínica Médica en agosto de 2004 (60\% sexo femenino; edad promedio: 60,9 $\pm 23,1$ años; media de internación: 5,8 $\pm 2,7$ días). Los diagnósticos documentados en el primer día de hospitalización fueron manualmente transcritos y analizados según sus frecuencias. Fueron documentados 144 diagnósticos (31 categorías), media de 4,8 \pm 4,0 por paciente (variación $=1-10$ ) y los más frecuentes fueron: Dolor agudo (66,7\%), Deterioro de la integridad tisular (63,3\%), Limpieza inefectiva de las vías aéreas (43,3\%), Riesgo de deterioro de la integridad cutánea $(36,7 \%)$ y Deterioro de la integridad cutánea (33,3\%). Son presentados los resultados e intervenciones para los 3 más frecuentes.

\footnotetext{
* Extraído da tese "Efeitos da implementação de uma classificação diagnóstica na clínica médica do Hospital Universitário da Universidade de São Paulo", Escola de Enfermagem, Universidade de São Paulo (EEUSP), 2006

1 Enfermeira. Doutoranda, Programa de Pós-Graduação em Enfermagem na Saúde do Adulto da EEUSP. Enfermeira do Hospital de Reabilitação de Anomalias Craniofaciais, Universidade de São Paulo, Bauru, SP, Brasil. pccf@terra.com.br

2 Enfermeira. Professora Titular, EEUSP. São Paulo, SP, Brasil. mtmllf@usp.br
}

\section{KEY WORDS}

Nursing diagnoses. Classification. Internal medicine.

\section{DESCRIPTORES}

Diagnóstico de enfermería.

Clasificación.

Medicina interna. 


\section{INTRODUÇÃO}

\section{$O$ processo de enfermagem}

O processo de enfermagem (PE) consolida-se na prática clínica da enfermagem e orienta o trabalho do enfermeiro para coletar dados, identificar as necessidades de cuidados, propor intervenções e avaliar os resultados dos cuidados que realiza. A documentação do PE pode ser um instrumento útil para a avaliação do cuidado pelo gerenciamento das informações de enfermagem.

A fase do diagnóstico de enfermagem vem sendo introduzida no PE em vários serviços de enfermagem no Brasil. A inserção do diagnóstico de enfermagem requer que os enfermeiros tenham uma linguagem comum que favoreça 0 entendimento entre os seus pares sobre os fenômenos clínicos de interesse, norteando as decisões sobre o que fazer por eles. A contextualização do diagnóstico de enfermagem no PE pode ter efeitos positivos na definição dos fenômenos, na proposição de intervenções de enfermagem e avaliação dos resultados obtidos.

A implementação de uma classificação diagnóstica na prática clínica permite aos enfermeiros nomear com maior clareza os focos do cuidado pelos quais são responsáveis. Para tal nomeação é necessário que os enfermeiros envolvidos nesse processo compartilhem das mesmas idéias sobre as coisas pelas quais pensam ser responsáveis no processo de cuidar ${ }^{(1-2)}$.

A introdução da fase do diagnóstico de enfermagem no PE em qualquer serviço de enfermagem exige esforços para uma mudança planejada: preparação da equipe, realização de cursos, seminários, preparo para a informatização do serviço, acordo entre sujeitos da mudança sobre a linguagem comum que será utilizada por eles, criação de grupos facilitadores, reuniões mensais com a equipe e a diretoria do serviço ${ }^{(3)}$. Por isso constitui-se um grande desafio para todos da equipe de enfermagem. Os esforços de implementação de uma classificação de enfermagem nos serviços devem ser acompanhados de uma avaliação sistemática dos seus resultados, visando um contínuo planejamento para manutenção e refinamento da sua utilização.

\section{A Taxonomia II da NANDA- I}

A introdução da classificação diagnóstica da NANDA I na prática clínica dos enfermeiros necessita ter o sentido de que seu uso pode levar ao alcance de resultados almejados pelo planejamento da assistência.

A Taxonomia da NANDA-I, conforme a revisão de 2005, é estruturada em 13 domínios e 47 classes contendo 172 diagnósticos de enfermagem. Um domínio representa uma esfera de atividade, estudo ou interesse ${ }^{(4)}$. Ele reflete o conteúdo dos seus diagnósticos e representa a similaridade de seu contingente. Uma classe é uma subdivisão de um grupo maior, uma divisão de pessoas ou coisas por qualidade, grau ou categoria. Um diagnóstico de enfermagem consiste em

um julgamento clínico sobre a resposta de um indivíduo, uma família ou uma comunidade com relação a problemas de saúde reais ou potenciais/ processo de vida que fornecem a base para uma terapia definitiva que busca alcançar resultados nos quais a enfermagem é necessária( ${ }^{(4)}$.

A implementação dessa linguagem comum implica em acordo sobre o que os seus termos representam para quem a utiliza, em proposição de intervenções para os diagnósticos de enfermagem e verificação de resultados alcançados com as intervenções realizadas ${ }^{(5)}$.

Ligações entre os diagnósticos da NANDA-I e as intervenções da Classificação Intervenções de Enfermagem (Nursing Interventions Classification - NIC) relacionam um diagnóstico com uma ou mais intervenções de enfermagem para que se obtenham resultados possíveis e desejáveis para o paciente. Essas ligações facilitam a fundamentação diagnóstica e a tomada de decisão clínica pelo enfermeiro sobre os resultados desejáveis e sobre as intervenções para alcançá-los.

A conexão entre as linguagens padronizadas aqui referidas como NANDA, NIC e NOC e a sua harmonização é um grande passo para que os enfermeiros possam mapear $o$ território pelo qual têm responsabilidade ${ }^{(6)}$.

Considerando que, na seqüência dos trabalhos de implementação da classificação de diagnósticos num serviço é importante organizar informações que permitam planejar a manutenção e o refinamento do seu uso, e que a articulação entre os diagnósticos, intervenções e resultados são importantes para refinar o uso dos diagnósticos, este estudo teve os objetivos de: descrever os diagnósticos de enfermagem documentados por enfermeiros após 3 meses da implementação da classificação diagnóstica da NANDA I e propor ligações entre intervenções, resultados e os três diagnósticos de enfermagem mais freqüentes.

\section{MÉTODO}

É um estudo retrospectivo, subprojeto de estudo que trata de avaliar os efeitos da implementação de uma classificação diagnóstica na prática clínica do enfermeiro, que está sendo desenvolvido no Hospital Universitário da Universidade de São Paulo (HU-USP). O projeto foi aprovado pela Comissão de Ética em Pesquisa da Instituição.

A Clínica Médica do HU-USP foi a primeira unidade da Instituição em que foi implementada a documentação formal dos diagnósticos de enfermagem de acordo com taxonomia da NANDA-I (maio de 2004). Essa foi a unidade de origem dos pacientes cujos diagnósticos foram analisados neste estudo.

Definiu-se 30\% do total de internações como o número mínimo de prontuários a serem analisados. No mês de agosto 
de 2004 houve 87 internações na Clínica Médica. Da listagem oferecida pelo serviço de arquivo e estatística do HU-USP foram selecionados 30 prontuários (34\% do total das internações no mês na Clínica Médica). Os conteúdos dos registros dos diagnósticos de enfermagem nas primeiras 24 horas de internação foram transcritos em um instrumento contendo espaço adicional para a coleta dos dados de características dos pacientes. Foram também coletados dados de caracterização das enfermeiras que realizaram a documentação dos diagnósticos. A partir do nome e registro da enfermeira na ficha dos diagnósticos de enfermagem do paciente, buscaram-se os dados de caracterização das enfermeiras no Departamento de Enfermagem da Instituição.

Os diagnósticos documentados foram analisados em freqüências absolutas e relativas. A proposta de intervenções e resultados foi feita com base na $\mathrm{NOC}^{(5)}$ e na $\mathrm{NIC}^{(7)}$. A seleção das intervenções e resultados para cada um dos três diagnósticos mais freqüentes foi uma proposta das autoras deste trabalho com base na experiência clínica e nas sugestões das próprias classificações de intervenções e de resultados ${ }^{(5,7)}$.

Os pacientes cujos diagnósticos foram analisados eram predominantemente do sexo feminino (60\%), com idades entre
16 e 95 anos, média de 60,9 anos ( $\pm 23,1)$ e média de internação de $5,8( \pm 2,7)$ dias. Os diagnósticos médicos predominantes foram, de acordo com o CID-10 ${ }^{(8)}$, as Doenças do Aparelho Respiratório (30\%) e, quanto às condições de saída, 83,3\% tiveram alta hospitalar; $10 \%$ foram a óbito e 6,7\% foram transferidos para outro serviço. Nove enfermeiras fizeram as documentações dos diagnósticos nos prontuários selecionados; $100 \%$ eram mulheres, com idade média de 29,4( $\pm 6,4)$ anos, tempo de formada entre 1 e 10 anos e 2 tinham o mestrado como maior grau de formação. O tempo de experiência profissional variou entre 1 e 10 anos, e 44,4\% haviam participado do curso preparatório para a implementação da classificação no HU. Cada enfermeira fez os registros de 1 a 5 pacientes cujos prontuários foram analisados.

\section{RESULTADOS}

Para os 30 pacientes, no primeiro dia de internação, foram documentados 144 diagnósticos de enfermagem em 31 categorias diagnósticas. A média foi de 4,8( $\pm 4,0)$ diagnósticos, variando de 1 a 10 diagnósticos por paciente. A Tabela 1 mostra, segundo os domínios da classificação da NANDAI, as freqüências de pacientes que tiveram cada diagnóstico documentado.

Tabela 1 - Freqüência dos diagnósticos segundo os domínios da classificação da NANDA-I. (n=30) - São Paulo - 2005

\begin{tabular}{|c|c|c|c|c|}
\hline \multirow{2}{*}{ Domínio } & \multirow{2}{*}{ Categoria Diagnóstica } & & \multicolumn{2}{|c|}{ Freqüência } \\
\hline & & & $\mathbf{N}$ & $\%$ \\
\hline \multirow{9}{*}{ Segurança/Proteção } & - Integridade Tissular Prejudicada & & 19 & 63,3 \\
\hline & - Desobstrução Ineficaz de Vias Aéreas & & 13 & 43,3 \\
\hline & - Risco para Integridade da Pele Prejudicada & & 11 & 36,7 \\
\hline & - Integridade da Pele Prejudicada & & 10 & 33,3 \\
\hline & - Risco de Quedas & & 6 & 20,0 \\
\hline & - Hipertermia & & 4 & 13,3 \\
\hline & - Mucosa Oral Prejudicada & & 4 & 13,3 \\
\hline & - Risco de Aspiração & & 2 & 6,7 \\
\hline & & Sub-total & 76 & \\
\hline \multirow{10}{*}{ Atividade/Repouso } & - Padrão respiratório ineficaz & & 7 & 23,3 \\
\hline & - Mobilidade física prejudicada & & 6 & 20,0 \\
\hline & - Perfusão tissular ineficaz renal & & 5 & 16,7 \\
\hline & - Déficit no Autocuidado total & & 4 & 13,3 \\
\hline & - Perfusão tissular ineficaz cerebral & & 3 & 10,0 \\
\hline & - Perfusão tissular ineficaz periférica & & 2 & 6,7 \\
\hline & - Débito cardíaco diminuído & & 2 & 6,7 \\
\hline & - Déficit no Autocuidado para Banho/Higiene & & 1 & 3,3 \\
\hline & - Fadiga & & 1 & 3,3 \\
\hline & & Sub-total & 31 & \\
\hline
\end{tabular}


(continuação)

\begin{tabular}{|c|c|c|c|}
\hline \multirow{2}{*}{ Domínio } & \multirow{2}{*}{ Categoria Diagnóstica } & \multicolumn{2}{|c|}{ Freqüência } \\
\hline & & $\mathbf{N}$ & $\%$ \\
\hline \multirow{4}{*}{ Conforto } & - Dor aguda & 20 & 66,7 \\
\hline & - Náusea & 2 & 6,7 \\
\hline & - Dor crônica & 1 & 3,3 \\
\hline & Sub-total & 23 & \\
\hline \multirow{6}{*}{ Eliminação } & - Constipação & 2 & 6,7 \\
\hline & - Retenção urinária & 1 & 3,3 \\
\hline & - Eliminação Urinária prejudicada & 1 & 3,3 \\
\hline & - Diarréia & 1 & 3,3 \\
\hline & - Troca de gases prejudicada & 1 & 3,3 \\
\hline & Sub-total & 6 & \\
\hline Enfrentamento/ & Ansiedade & 4 & 13,3 \\
\hline Tolerância ao Estresse & Sub-total & 4 & \\
\hline \multirow{3}{*}{ Nutrição } & $\begin{array}{l}\text { - Risco para nutrição desequilibrada: menos do que as necessidades } \\
\text { corporais }\end{array}$ & 1 & 3,3 \\
\hline & - Volume excessivo de líquidos & 1 & 3,3 \\
\hline & Sub-total & 2 & \\
\hline \multirow{3}{*}{ Percepção/Cognição } & Confusão aguda & 1 & 3,3 \\
\hline & Confusão crônica & 1 & 3,3 \\
\hline & Sub-total & 2 & \\
\hline
\end{tabular}

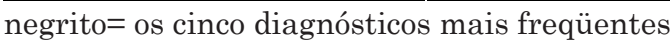

Os diagnósticos mais freqüentes foram: Dor aguda (66,7\%), Integridade tissular prejudicada (63,3\%) Desobstrução Ineficaz de vias aéreas (43,3\%), Risco para integridade da pele prejudicada (36,7\%), Integridade da pele prejudicada (33,3\%).

Consideramos os domínios da Taxonomia II e os diagnósticos que os compõem. Dos 13 domínios, 5 não foram representados por nenhum diagnóstico do seu grupo: Promoção à Saúde, Autopercepção, Relacionamento de Papel, Sexualidade e Crescimento e Desenvolvimento.

Os domínios Nutrição, Eliminação, Atividade/Repouso, Percepção/Cognição, Enfrentamento/Tolerância ao Estresse,
Segurança/proteção e Conforto foram representados pela ocorrência de pelo menos um de seus respectivos diagnósticos. Os domínios mais representados foram os de Segurança/ Proteção, com 76 diagnósticos e de Atividade/Repouso, com 31 diagnósticos. Esses dois domínios compreenderam 74\% (107) dos diagnósticos documentados pelas enfermeiras.

Os três diagnósticos mais freqüentes (Dor Aguda/66,7\%; Integridade Tissular Prejudicada/63,3\% e Desobstrução Ineficaz de Vias Aéreas/43,3\%) são descritos a seguir com suas respectivas características definidoras, de acordo com o registro realizado pelos enfermeiros da Clínica Médica. Os fatores relacionados dos diagnósticos de enfermagem não foram apontados nos registros das enfermeiras.

Tabela 2 - Diagnósticos de Enfermagem mais freqüentes e suas características definidoras - São Paulo - 2005

\begin{tabular}{|c|c|c|c|}
\hline Diagnóstico de Enfermagem & Característica Definidora & $\mathbf{N}$ & $\%$ \\
\hline \multirow[b]{2}{*}{ Dor $(n=20)$} & - relato verbal & 16 & 80,0 \\
\hline & - evidência observada & 5 & 25,0 \\
\hline \multirow{4}{*}{ Integridade Tissular Prejudicada $(n=19)$} & - tecido lesado & 11 & 57,8 \\
\hline & - equimose & 10 & 52,6 \\
\hline & - hiperemia & 3 & 15,7 \\
\hline & - necrose tecidual & 1 & 5,3 \\
\hline \multirow{5}{*}{$\begin{array}{l}\text { Desobstrução Ineficaz de Vias Aéreas } \\
(\mathrm{n}=12)\end{array}$} & - ruídos adventícios & 7 & 58,3 \\
\hline & - dispnéia & 7 & 58,3 \\
\hline & - tosse ineficaz & 6 & 50,0 \\
\hline & - estertores creptantes & 4 & 33,3 \\
\hline & - roncos & 1 & 8,3 \\
\hline
\end{tabular}




\section{Proposta de resultados e intervenções da $\mathrm{NOC}^{(5)}$ e da NIC(7)}

A atual classificação da NOC é uma lista de 260 resultados de enfermagem com definições, indicadores e escalas de medidas ${ }^{(5)}$. Os resultados podem ser usados para indivíduos, famílias e comunidades. Os resultados representam estados do paciente/cliente que podem variar, ser mensurados e comparados a dados iniciais ao longo do tempo do tratamento de enfermagem e após as intervenções propostas. Eles definem um estado do paciente em determinado momento do tempo e podem indicar melhora ou piora ${ }^{(5)}$.

As intervenções de enfermagem descrevem os comportamentos dos enfermeiros mediante a preocupação de obter resultados dos pacientes a partir dos fenômenos tratáveis pelo enfermeiro, os diagnósticos de enfermagem ${ }^{(5)}$.

Os resultados e as intervenções são selecionados em relação a determinado diagnóstico de enfermagem. A intervenção está voltada para a alteração dos fatores relacionados dos diagnósticos de enfermagem da NANDA- ${ }^{(1)}$ ou de qualquer outra classificação diagnóstica que se esteja usando. Se a intervenção é bem sucedida em alterar os fatores relacionados, pode-se esperar a melhora do estado do paciente. Nem sempre é possível mudar os fatores relacionados, e se assim for, é necessário focalizar as intervenções nas características definidoras ${ }^{(1,7)}$.

Cada intervenção possui um código específico com quatro dígitos. As atividades relacionadas às intervenções não possuem códigos, mas são codificáveis, se preciso for. Nem todas as atividades pertencentes a uma determinada intervenção são necessariamente utilizadas quando se faz a escolha da intervenção $0^{(7)}$.

A $\mathrm{NOC}^{(5)}$ faz uso de indicadores de resultado de enfermagem para descrever estados específicos do paciente que podem ser sensíveis às intervenções de enfermagem. Esses indicadores podem ser definidos como medidas para avaliar os resultados das intervenções. A avaliação de resultado de enfermagem é a operação que envolve decidir o melhor indicador de resultado a ser utilizado, a forma e o tempo em que será quantificado ${ }^{(5)}$.

Apresentamos a seguir as propostas de resultados e intervenções para os três diagnósticos mais freqüentes com base nas características definidoras dos diagnósticos, pois não houve descrições dos fatores relacionados nos registros encontrados na amostra do estudo. Os códigos numéricos citados entre parênteses são os designados pelas classificações da $\operatorname{NOC}^{(5)} \mathrm{e}$ NIC ${ }^{(7)}$ para especificar os resultados e intervenções.

Quadro 1 - Intervenções e resultados de enfermagem para o diagnóstico de dor - São Paulo - 2005

\begin{tabular}{|ll|}
\hline \multicolumn{1}{|c|}{$\begin{array}{c}\text { Resultados e indicadores } \\
\text { (código NOC(5)) }\end{array}$} & \multicolumn{1}{c|}{$\begin{array}{c}\text { Intervenções e atividades } \\
\text { (código NIC(7)) }\end{array}$} \\
\hline - Nível de dor (2102): & - Controle da dor (1400) \\
Dor relatada (210201) & Realizar levantamento sobre as características, \\
Expressões orais e faciais de dor (210202) & local, início intensidade, freqüência, a qualidade, \\
Inquietação (210208) & intensidade, gravidade da dor e fatores \\
- Nível de conforto (2100): & precipitantes; \\
Bem estar físico relatado (210001) & Observar indicadores não verbais de desconforto; \\
Satisfação expressa com o controle da dor (210008) & Assegurar cuidados precisos de analgesia \\
Freqüência da dor (210203) & Avaliar eficácia de medidas de controle da dor. \\
\hline
\end{tabular}

Quadro 2 - Intervenções e resultados de enfermagem para o diagnóstico de integridade tissular prejudicada - São Paulo - 2005

\begin{tabular}{|cc|}
\hline $\begin{array}{c}\text { Resultados e indicadores } \\
\text { (código NOC(5)) }\end{array}$ & \multicolumn{1}{c|}{$\begin{array}{c}\text { Intervenções e atividades } \\
\text { (código NIC(7)) }\end{array}$} \\
\hline - Integridade tissular/pele e mucosas (1101): & - Cuidados com lesões (3660) \\
Temperatura (110101); & Massagear área em torno da lesão para estimular circulação; \\
Pigmentação (110105); & manter técnica asséptica no curativo ao fazer os cuidados da \\
Cor (110107); & lesão; examinar a ferida a cada troca de curativo; observar \\
Textura (110108); & as características da lesão; administrar medicamentos \\
Perfusão tissular (110111); & tópicos se necessário; examinar/observar monitorar as \\
Integridade da pele (110113); & condições locais da pele como ruptura, cor, calor, textura, \\
- Cicatrização de feridas (1102): & edemas, ulcerações, pontos de pressão, infecção, áreas de \\
Resolução de eritema (110208); & necrose, fontes de pressão e fricção; \\
- Cicatrização de feridas (1103): & \\
Resolução de necrose (110312); & \\
\hline
\end{tabular}


Quadro 3 - Intervenções e resultados de enfermagem para o diagnóstico de desobstrução ineficaz de vias aéreas - São Paulo - 2005

\begin{tabular}{|c|c|}
\hline $\begin{array}{l}\text { Resultados e indicadores } \\
\text { (código } \mathrm{NOC}^{(5)} \text { ) }\end{array}$ & $\begin{array}{l}\text { Intervenções e atividades } \\
\left.\text { (código } \mathrm{NIC}^{(7)}\right)\end{array}$ \\
\hline \multicolumn{2}{|r|}{ - Controle das vias aéreas (3140): } \\
\hline - Estado respiratório: desobstrução de vias aéreas (0410): & Remover secreções, encorajando o tossir e a aspiração; \\
\hline Taxa e ritmo respiratório (041004); & orientar sobre as maneiras de tossir eficientemente; \\
\hline Aspiração traqueal (041006); & auscultar os sons respiratórios, observando as áreas de \\
\hline \multirow[t]{3}{*}{ Livre de sons respiratórios estranhos (041007) } & ventilação diminuídas ou ausentes e presença de ruídos \\
\hline & adventícios; posicionar paciente de modo a aliviar a \\
\hline & dispnéia e maximizar o potencial ventilatório \\
\hline
\end{tabular}

\section{DISCUSSÃO}

Neste estudo descrevemos a freqüência dos diagnósticos de enfermagem apontados nos registros feitos por enfermeiras no ato da admissão de pacientes e propusemos resultados e intervenções de enfermagem para os três mais freqüentes.

As 31 categorias de diagnósticos refletem os fenômenos que os enfermeiros estavam focalizando na admissão dos pacientes da amostra na clínica médica. Observamos que quatro dos cinco diagnósticos mais freqüentes pertencem ao Domínio Segurança/Proteção. Esse domínio tem como definição: Estar livre de perigo, lesão física ou dano do sistema imunológico, preservação contra perda e proteção da segurança e seguridade ${ }^{(4)}$. Os diagnósticos de enfermagem mais freqüentes no estudo, pertencentes ao Domínio Segurança/Proteção, foram: Integridade tissular prejudicada e desobstrução ineficaz de vias aéreas e pertencentes à Classe 2 - Dano ou ferimento corporal.

O diagnóstico de Integridade Tissular Prejudicada tem como definição: Dano às membranas mucosas, córnea, pele ou tecidos subcutâneos ${ }^{(4)}$ e foi representado neste estudo pelas seguintes características definidoras: equimose, tecido lesado, hematoma, hiperemia e necrose tecidual. São sinais de sofrimento tissular e que podem ser advindos de vários fatores causais como imobilização no leito, diminuição de atividade motora, falta de adequada perfusão sanguínea tissular. Porém, os fatores relacionados do diagnóstico não foram apontados nos registros transcritos. Os fatores que poderiam estar contribuindo para essa resposta incluem: punção venosa periférica, torácica, dificuldades de mobilização do paciente, assim como a prevenção de lesões de pele/tecidos e mobilização precoce no leito. Por se tratar de amostra de pacientes de clínica médica as incisões cirúrgicas, provavelmente não são fatores relacionados freqüentes.

Em estudo realizado nos Estados Unidos ${ }^{(9)}$, a análise de 477.000 registros eletrônicos de pacientes hospitalizados mostrou que o número de diagnósticos de enfermagem por paciente variou de 1 a 11, com média de 3,4 diagnósticos por paciente e os mais freqüentes foram: Potencial para Integridade da Pele Prejudicada, Déficit de Autocuidado banho/ higiene e Integridade da Pele Prejudicada. Os diagnósticos referentes às alterações de pele foram também os mais freqüentes no presente estudo. Observamos que, freqüentemente, os diagnósticos de integridade da pele prejudicada e de integridade tissular prejudicada são aplicados nas mesmas situações. Na instituição em que o presente estudo foi realizado, as enfermeiras aplicam o de integridade tissular prejudicada quando já houve solução de continuidade em tecido subcutâneo. Não é raro observarmos a aplicação de prejuízo da integridade de pele também nessas situações, o que pode ter acontecido no estudo citado $^{(9)}$, fazendo os seus resultados, em termos de diagnósticos mais freqüentes, semelhantes aos do presente estudo.

Em estudo realizado em uma unidade médico cirúrgica de um hospital da cidade de Londrina ${ }^{(10)}$ foram identificados em 60 pacientes 338 diagnósticos de enfermagem, com 49 categorias diagnósticas diferentes e com média de 5,6 diagnósticos por paciente, variando de 1 a 13 para cada um deles. Os cinco diagnósticos de enfermagem mais freqüentes foram: Risco de Infecção (58,3\%), Dor (50\%), Constipação (41,6\%), Intolerância à Atividade (35\%) e Distúrbio do Padrão do Sono (28,3\%). A amostra desse estudo ${ }^{(10)}$ tem semelhança com a do presente estudo, mas não foram identificadas entre as categorias diagnósticas mais freqüentes, categorias de Integridade Tissular Prejudicada (risco ou atual), o que poderíamos esperar no perfil de pacientes de uma clínica médico-cirúrgica. No presente estudo o diagnóstico de Dor teve freqüência de $66,7 \%$ (Tabela 1), um pouco mais elevada que a encontrada no estudo citado ${ }^{(10)}$, confirmando que essa resposta deve ser foco freqüente de atenção do enfermeiro. Programas de capacitação do enfermeiro para o alívio da dor são importantes para a qualidade da assistência de enfermagem.

O diagnóstico de Desobstrução Ineficaz de Vias Aéreas tem como definição: incapacidade de eliminar secreções ou obstruções do trato respiratório para manter uma via aérea desobstruída ${ }^{(4)}$ e foi representado pelas seguintes características definidoras: ruídos adventícios, dispnéia, 
tosse ineficaz, estertores creptantes e roncos. Era esperado que esse fenômeno fosse característico da amostra do estudo, por se tratar de pacientes adultos internados em clínica médica, destacando-se que houve predominância de internações por doenças do aparelho respiratório.

O Domínio Conforto foi representado pelo diagnóstico de dor aguda, com a maior freqüência e pertencente à Classe 1 - Conforto Físico. Esse domínio tem como definição: Sensação de bem-estar ou conforto mental, físico ou social ${ }^{(4)}$. O diagnóstico de Dor Aguda tem como definição:

Experiência sensorial e emocional desagradável que surge de lesão tissular real ou potencial ou descrita em termos de tal lesão (Associação Internacional para o estudo da Dor); início súbito ou lento, de intensidade leve a intensa, com término antecipado ou previsível e duração de menos de seis meses.

Foi descrito aqui pelas seguintes características definidoras: relato verbal ou evidência observada. O empenho para aquisição de habilidades na identificação dos fatores relacionados são requisitos para propor intervenções de enfermagem cada vez mais eficazes, principalmente para obtenção de resultados que proporcionem conforto e alívio do fenômeno assim como a promoção do autocuidado e autoconhecimento sobre controle da analgesia e de terapias alternativas para alívio e controle da ansiedade.

A escolha dos resultados e seus indicadores para nortear a proposição das intervenções de enfermagem depende das habilidades clínicas do enfermeiro, especialmente das envolvidas na acurácia diagnóstica ${ }^{(11)}$. Um diagnóstico de enfermagem pode ser mais ou menos acurado ${ }^{(6)}$. Diagnósticos pouco acurados podem culminar com a realização de intervenções desnecessárias ou mesmo danosas para o paciente, prejudicando a qualidade do cuidado. Por isso, a preocupação com a acurácia dos diagnósticos estabelecidos pelos enfermeiros deve estar entre as prioridades dos serviços de enfermagem $^{(12)}$. A escolha dos resultados e intervenções para cada diagnóstico também depende das habilidades clínicas do enfermeiro, que deve considerar as peculiaridades de cada situação, as preferências de cada paciente para definir os resultados possíveis e desejáveis e, depois, as intervenções para alcançá-los. As classificações $\mathrm{NOC}^{(5)}$ e NIC ${ }^{(7)}$ apresentam sugestões de ligações entre diagnósticos, intervenções e resultados que podem ajudar o enfermeiro que tem pouca familiaridade com as classificações ou com determinados diagnósticos.

\section{CONCLUSÃO}

Em relação ao objetivo proposto, de descrever os diagnósticos de enfermagem documentados após 3 meses de implantação da classificação diagnóstica da NANDA-I, dos 13 domínios da Classificação, 7 foram representados pelos seus respectivos diagnósticos de enfermagem (Tabela 1). O domínio de Segurança/proteção foi o que mais obteve representatividade pelos seus respectivos diagnósticos. Os três diagnósticos mais freqüentes foram Dor Aguda, Integridade Tissular Prejudicada e Desobstrução Ineficaz de Vias Aéreas. Eles caracterizam as necessidades de cuidados mais freqüentes focalizadas pelos enfermeiros que avaliaram os pacientes na sua admissão na unidade.

Em relação à proposta de intervenções e resultados para os três diagnósticos mais freqüentes, sugere-se que a documentação dos fatores relacionados dos diagnósticos, além das características definidoras, proporcionaria refinamento dos registros, permitindo melhor análise de seus conteúdos. A disponibilidade de registros de boa qualidade permite estimar a qualidade do cuidado e permite a realização de pesquisas de efetividade do cuidado.

Os resultados deste estudo representam parte dos esforços de um grupo de enfermeiros interessados em promover o avanço da enfermagem clínica. Os diagnósticos aqui estudados foram os documentados após três meses da implementação da classificação da NANDA-I. O uso continuado dos diagnósticos na prática clínica traz constantes desafios e também é com o próprio uso que os enfermeiros vão ajustando os significados dos enunciados diagnósticos nas situações em que os aplicam. Com o tempo é possível que algumas freqüências de diagnósticos se ajustem em decorrência de melhor compreensão sobre a aplicação de determinados termos e não só como resultado de mudanças nas situações dos pacientes.

Os resultados de estudos como este são importantes para a organização de conteúdos de ensino para alunos e enfermeiros preparando-se para cuidar de pacientes em áreas específicas. Conhecendo os diagnósticos mais freqüentes eles podem se preparar para realizar avaliações direcionadas para identificar esses diagnósticos e os resultados a eles relacionados, assim como para realizar as intervenções para atingi-los. 


\section{REFERÊNCIAS}

1. Cianciarullo TI, Gualda DMR, Melleiro MM, Anakubi MI, organizadoras. Sistema de Assistência de Enfermagem: evolução e tendências. $3^{\text {a }}$ ed. São Paulo: Ícone; 2005.

2. Hayakawa SI, Hayakawa SI. Language in thought and action. $5^{\text {th }}$ ed. San Diego: Harcourt Brace; 1990.

3. Lima AFC. Significado que as enfermeiras assistenciais de um hospital universitário atribuem ao processo de implementação do diagnóstico de enfermagem como etapa do Sistema de Assistência de Enfermagem - SAE [tese]. São Paulo: Escola de Enfermagem, Universidade de São Paulo; 2004.

4. North American Diagnoses Association (NANDA). Nursing diagnoses: definitions \& classification: 2005-2006. Philadelphia; 2005.

5. Johnson M, Maas M, Moorhead S. Nursing Outcomes Classification (NOC). St. Louis: Mosby; 2000.

6. Lunney M. Theoretical explanations form combining NANDA, NIC and NOC. In: Dochterman JM, Jones DA. Unifying nursing languages: the harmonization of NANDA, NIC and NOC. Washington: American Nurses Association; 2003. p. 35-45.
7. McCloskey JC, Bulechek GM. Nursing Interventions Classifications (NIC): Iowa intervention project. St. Louis: Mosby; 2000.

8. Organização Mundial da Saúde (OMS). Classificação Estatística Internacional de Doenças e Problemas Relacionados à Saúde: CID 10. São Paulo: EDUSP; 1998.

9. Delaney C, Reed D, Clark M. Describing patient problems \& nursing treatment patterns using nursing minumum data sets (NMDS \& NMMDS) \& UHDDS repositories. Proc AMIA Symp. 2000:176-9.

10. Volpato MP. Diagnósticos de enfermagem em clientes internados em unidade médico-cirúrgica [dissertação]. São Paulo: Escola de Enfermagem, Universidade de São Paulo; 2001.

11. Lunney M. Critical thinking and accuracy of nurses' diagnoses. Part I: Risk of low accuracy diagnoses and new views of critical thinking. Rev Esc Enferm USP. 2003;37(2):17-27.

12. Lunney M. Critical thinking and accuracy of nurses' diagnoses. Part II: Application of cognitive skills and guidelines for selfdevelopment. Rev Esc Enfem USP. 2003;37(3):106-12. 